\title{
Schunk classes are nilpotent product closed
}

\section{James Wiegold}

The following result is proved. Let $\underline{X}$ be a Schunk class and $k$ a positive integer. Then the $k$-th nilpotent product of any two groups in $\underline{X}$ is in $\underline{X}$.

Let $\underline{X}$ be a class of finite soluble groups. Then $\underline{X}$ is a Schunk class if the following two conditions hold.

(i) Every epimorphic image of a group in $\underline{X}$ is in $\underline{X}$.

(ii) Let $G$ be a finite soluble group such that every epimorphic image of $G$ having a faithful primitive permutation representation is in $\underline{X}$. Then $G$ is in $\underline{X}$.

In his lectures to the Summer Research Institute of the Australian Mathematical Society at Canberra in January 1969, W. Gaschütz gave a proof of the fact that Schunk classes are direct product closed, using a lemma of Itô about maximal subgroups of a direct product. Here we offer a proof of a more general result:

THEOREM. Let $\underline{X}$ be a Schunk class and $k$ a positive integer. Then the $k$-th nilpotent product of any two groups in $\underline{X}$ is in $\underline{X}$.

Proof. Let $A_{1}$ and $B_{1}$ be any groups in $\underline{X}$ and $G_{1}$ their $k$-th nilpotent product. We shall show that every primitive epimorphic image of $G_{1}$ is an epimorphic image of $A_{1}$ or of $B_{1}$, and that will be more than enough to ensure that $G_{1}$ is in $\underline{X}$. Let $G$ be an epimorphic image of $G_{1}$ which is a primitive permutation group on a set $\Omega$, and let $A$ and $B$ stand for the images of $A_{1}$ and $B_{1}$ under the epimorphism. If $A$ or $B$ 
is 1 , then $G$ is $B$ or $A$; so we may assume that $A$ and $B$ are both non-trivial. In this situation it will appear that $G$ is cyclic of prime order so that we have the stronger conclusion that $G=A=B$.

Firstly suppose that the mutual commutator subgroup $[A, B]$ is not 1 . Then it follows from the definition of $k$-th nilpotent product (see [1]) that $[A, B]$ contains non-trivial elements of the centre of $G$. But then a subgroup $Z$ of prime order in the centre of $G$ is transitive on $\Omega$ since it is normal in $G$ ([2], Theorem 8.8), and regular since it is transitive and abelian. This means that $G=Z$, otherwise $G$ would contain abelian subgroups containing $Z$ strictly, which is evidently not possible.

Finally suppose that $[A, B]=1$. Then $A$ and $B$ are normal in $G$ since they generate $G$; and they are therefore transitive on $\Omega$. However, by ([2], Exercise 4.5'), the centralizer of a transitive subgroup is semiregular, which yields in our case that $A$ and $B$ are both regular. Since $G$ is soluble (and this is the one point at which solubility is used) it is monolithic, and the monolith $M$ is regular and abelian. As normal subgroups of $G, A$ and $B$ must contain $M$, so that $A=B=M$ since these three groups are of the same order. We have proved that $G=M$; and, as in the preceding paragraph, we conclude that $G$ is of prime order. This completes the proof.

\section{References}

[1] O.N. Golovin, "Nilpotent products of groups", Amer. Math. Soc. Transt. (2) 2 (1956), 89-115. Translated from Mat. Sb. N.S. 27 (69) (1950), 427-454.

[2] Helmut Wielandt, Finite permutation groups (Academic Press, New York, $1964)$.

Department of Pure Mathematics, SGS,

Australian National University,

Canberra. A.C.T.

Department of Pure Mathematics, University College,

Cardiff, U.K. 